\title{
NEW PARAMETER FOR DESCRIBING AND ANALYZING THE OPTICAL-ANISOTROPIC PROPERTIES OF BIOLOGICAL TISSUES
}

\author{
Yu. A. USHENKO ${ }^{*}$, O. I. TELENGA ${ }^{\dagger}$, A. P. PERESUNKO* \\ and O. K. NUMAN* \\ *Correlation Optics Department, Chernivtsi National University \\ 2 Kotsyubinsky Street, Chernivtsi 58012, Ukraine \\ $\dagger$ Optics and Spectroscopy Department \\ Chernivtsi National University, 2 Kotsyubinsky Street \\ Chernivtsi 58012, Ukraine \\ \$Chernivtsi Medical University \\ 2 Theatral Sq, Chernivtsi 58002, Ukraine \\ \$yuriyu@gmail.com
}

Accepted 28 March 2011

\begin{abstract}
To characterize the degree of similarity inherent to parameters of the optically uniaxial birefringent protein-fibril networks of biological tissues, a new parameter - complex degree of mutual anisotropy - has been offered. The technique of polarization measuring the coordinate distributions of the complex degree of mutual anisotropy of biological tissues has been developed. It has been shown that statistical approach to the analysis of complex degree of mutual anisotropy distributions for biological tissues in various morphological and physiological states and for different optical thicknesses appears to be more sensitive and efficient in differentiation of physiological state, as compared to investigations of complex degree of mutual polarization in the corresponding laser images.
\end{abstract}

Keywords: Polarization; birefringence; correlation; statistics; biological tissue; skin derma.

\section{Introduction}

Historically, the optical methods of biological tissue investigations can be separated into three groups:

- The spectral photometric methods ${ }^{1-3}$ based on analysis of spatial $(r)$ or time $(\tau)$ changes of radiation field intensity, scattered by biological tissues;

- Polarization methods based on usage of the coherency matrix for complex amplitudes $\{\mathrm{K}(r, \tau)\}$ (see Refs. 4-6).

$\mathrm{K}(r, \tau)=\left\|\begin{array}{ll}\left\langle U_{x}(r, \tau) U_{x}^{*}(r, \tau)\right\rangle & \left\langle U_{x}(r, \tau) U_{y}^{*}(r, \tau)\right\rangle \\ \left\langle U_{x}^{*}(r, \tau) U_{y}(r, \tau)\right\rangle & \left\langle U_{y}(r, \tau) U_{y}^{*}(r, \tau)\right\rangle\end{array}\right\|$

and the analysis of the degree of polarization $P(r)$ as the correlation factor for complex orthogonal components of electromagnetic fluctuations $U_{x}, U_{y}$ 
in one of the points $(r)$ of the scattered radiation field $^{7}$

$$
P(r)=\sqrt{1-\frac{4 \mid\left[\left\langle U_{x}(r, \tau) U_{x}^{*}(r, \tau)\right\rangle\left\langle U_{y}(r, \tau) U_{y}^{*}(r, \tau)\right\rangle\right.}{\left[\left\langle U_{x}(r, \tau) U_{x}^{*}(r, \tau)\right\rangle+\left\langle U_{y}(r, \tau) U_{y}^{*}(r, \tau)\right\rangle\right]^{2}}} .
$$

- The correlation methods based on the analysis of the correlation degree $J$ for the parallel polarization components $U_{x}\left(r_{1}\right), U_{x}\left(r_{2}\right)$ of light fluctuations in various points of object field $\left(r_{1}, r_{2}\right)$ (see Refs. 7 and 8)

$$
J=\frac{\left\langle U_{x}\left(r_{1}, \tau\right) U_{x}^{*}\left(r_{2}, \tau\right)\right\rangle-\left\langle U_{x}^{*}\left(r_{1}, \tau\right) U_{x}\left(r_{2}, \tau\right)\right\rangle}{\left\langle U_{x}\left(r_{1}, \tau\right) U_{x}^{*}\left(r_{2}, \tau\right)\right\rangle+\left\langle U_{x}^{*}\left(r_{1}, \tau\right) U_{x}\left(r_{2}, \tau\right)\right\rangle} .
$$

For the complex analysis of polarizationally heterogeneous laser radiation fields, a new approach was offered in Refs. 9-16. It is based on generalization of the coherency matrix $\{\mathrm{K}(r, \tau)\}$ by substituting it with the polarization coherency matrix $\left\{\Phi\left(r_{1}, r_{2}, \tau\right)\right\}$ for two points $\left(r_{1}, r_{2}\right)$

$$
\{\mathrm{K}(r, \tau)\} \Rightarrow\left\{\Phi\left(r_{1}, r_{2}, \tau\right)\right\} .
$$

In the expanded form, the expression (4) can be rewritten as follows

$$
\begin{aligned}
& \left\|\begin{array}{ll}
\left\langle U_{x}(r, \tau) U_{x}^{*}(r, \tau)\right\rangle & \left\langle U_{x}(r, \tau) U_{y}^{*}(r, \tau)\right\rangle \\
\left\langle U_{y}(r, \tau) U_{x}^{*}(r, \tau)\right\rangle & \left\langle U_{y}(r, \tau) U_{y}^{*}(r, \tau)\right\rangle
\end{array}\right\| \\
& \Rightarrow\left\|\begin{array}{ll}
\left\langle U_{x}\left(r_{1}, \tau\right) U_{x}^{*}\left(r_{2}, \tau\right)\right\rangle & \left\langle U_{x}\left(r_{1}, \tau\right) U_{y}^{*}\left(r_{2}, \tau\right)\right\rangle \\
\left\langle U_{y}\left(r_{1}, \tau\right) U_{x}^{*}\left(r_{2}, \tau\right)\right\rangle & \left\langle U_{y}\left(r_{1}, \tau\right) U_{y}^{*}\left(r_{2}, \tau\right)\right\rangle
\end{array}\right\| .
\end{aligned}
$$

Here, \langle\rangle means the averaging operation over the time $\tau$ of orthogonal components $U_{x}, U_{y}$ of electromagnetic wave complex amplitude in the points with coordinates $\left(r_{1}, r_{2}\right)$.

In Ref. 17, to characterize the consistency between the polarization states of the object field in the points $\left(r_{1}, r_{2}\right)$ with the intensities $I\left(r_{1}, \tau\right)$, $I\left(r_{2}, \tau\right)$, a new parameter - complex degree of mutual polarization (CDMP) $V\left(r_{1}, r_{2}, \tau\right)$ - was introduced. It has the following analytical form:

$$
V\left(r_{1}, r_{2}, \tau\right)=4 \frac{\nu_{1}^{2}+\nu_{2}^{2}+\nu_{3}^{2}}{I\left(r_{1}, \tau\right) I\left(r_{2}, \tau\right)},
$$

where $\nu_{i}$ are the coefficients formed by combinations of the elements of the polarization coherency matrix

$$
\begin{aligned}
& \left\{\Phi\left(r_{1}, r_{2}, \tau\right)\right\} \\
& \nu_{1}=\frac{\left\langle U_{x}\left(r_{1}, \tau\right) U_{x}^{*}\left(r_{2}, \tau\right)\right\rangle-\left\langle U_{y}\left(r_{1}, \tau\right) U_{y}^{*}\left(r_{2}, \tau\right)\right\rangle}{2}, \\
& \nu_{2}=\frac{\left\langle U_{x}\left(r_{1}, \tau\right) U_{y}^{*}\left(r_{2}, \tau\right)\right\rangle+\left\langle U_{y}\left(r_{1}, \tau\right) U_{x}^{*}\left(r_{2}, \tau\right)\right\rangle}{2}, \\
& \nu_{3}=i \frac{\left\langle U_{x}\left(r_{1}, \tau\right) U_{y}^{*}\left(r_{2}, \tau\right)\right\rangle-\left\langle U_{y}\left(r_{1}, \tau\right) U_{x}^{*}\left(r_{2}, \tau\right)\right\rangle}{2} .
\end{aligned}
$$

These elements are determined as the difference of the values of visibility of interference images formed by electromagnetic waves from the points $r_{1}, r_{2}$. Measurements of these parameters were performed for different orientations of the polarization filter placed before the photodetector. In this case, the value $\nu_{1}$ is determined by the difference of contrast values for the rotation angles of the analyzer transmission plane $0^{\circ}, 90^{\circ}$ to the incidence plane; $\nu_{2}$ - for angles $45^{\circ}, 135^{\circ} ; \nu_{3}$ - for rotation of the axis of maximum velocity of quarter-wave plates at the angle $+45^{\circ}$ (right circular) and $-45^{\circ}$ (left circular).

It is easy to show that the expression for the CDMP in the case of laser radiation scattered stationary field takes the following form

$$
V\left(r_{1}, r_{2}\right)=\frac{\left(U_{x}\left(r_{1}\right) U_{x}^{*}\left(r_{2}\right)+U_{y}\left(r_{1}\right) U_{y}^{*}\left(r_{2}\right)\right)^{2}}{I\left(r_{1}\right) I\left(r_{2}\right)} .
$$

This "two-point" theoretical approach [(4)-(8)] was extended to the analysis of polarizationinhomogeneous laser images of human biological tissues with the aim of experimental diagnostics of optical anisotropic structure. ${ }^{18}$ In Ref. 19 the method of direct polarization measurement of the real part of CDMP for different points $\left(r_{1}, r_{2}\right)$ of optically thin image (extinction coefficient $\tau \leq 0.1$ ) of biological tissue layers was proposed

$$
\begin{aligned}
\operatorname{Re}\{V\} & \equiv \tilde{V}\left(r_{1}, r_{2}\right) \\
& =\frac{\left\{U_{x}\left(r_{1}\right) U_{x}\left(r_{2}\right)-U_{y}\left(r_{1}\right) U_{y}\left(r_{2}\right) \cos \left[\varphi\left(r_{1}\right)-\varphi\left(r_{2}\right)\right]\right\}^{2}}{I\left(r_{1}\right) I\left(r_{2}\right)},
\end{aligned}
$$

where $\varphi\left(r_{1}\right)$ and $\varphi\left(r_{2}\right)$ are the phase shifts between the orthogonal components $U_{x}, U_{y}$ of laser beam amplitude.

The ranges of changes of the 1st- to 4th-order distribution statistic moments $\tilde{V}(x, y)$ for the corresponding laser images important for diagnostics of the human connective tissue oncological state were 
determined in Refs. 19 and 20. On the other hand, these analytical techniques with account of $\tilde{V}(x, y)$ lead to disregarding the extracellular matrix birefringence of the biological tissues, which is a principal physical mechanism providing formation of their polarization-inhomogeneous images. ${ }^{8}$ That is why, it appears to be important to search for new diagnostic parameters directly characterizing the degree of consistency of optical axes and birefringence orientations of various points of protein-fibril network forming the biological tissue extracellular matrix. ${ }^{18}$

This work is aimed at substantiation of the technique of polarization-correlation mapping of the biological tissue birefringent networks for diagnostics of their physiological and pathological states.

\section{Theoretical Analysis of Laser Radiation Parameters Transformation by the Network of Optically Uniaxial Birefringent Protein Crystals}

The processes of laser beam interaction with biological tissues are discussed in the following model approximation $^{21-25}$ :

(1) According to the classification of Cowin, ${ }^{18}$ morphologically all varieties of human tissues consist of four main types (connective, muscle, epithelial and nervous), each of it is formed by a twocomponent amorphous-crystalline structure.

(2) The crystalline component of the biological tissues (extracellular matrix) is formed by a network of coaxial cylindrical protein (collagen, elastin, myosin, etc.) fibrils. ${ }^{18}$

(3) From the optical viewpoint, protein fibrils possess properties of optically uniaxial crystals with the index of birefringence $\Delta n$. Parameters of anisotropy $[\rho(r), \delta(r)]$ of these fibrils in every point $(r)$ are described by the Jones operator ${ }^{4}$ :

$$
\begin{aligned}
\{D\} & =\left\|\begin{array}{cc}
d_{11}(r) & d_{12}(r) \\
d_{21}(r) & d_{22}(r)
\end{array}\right\| \\
& =\left\|\begin{array}{c}
\cos ^{2} \rho(r)+\sin ^{2} \rho(r) \exp (-i \delta(r)) \\
\cos \rho(r) \sin \rho(r)[1-\exp (-i \delta(r))] \\
\cos \rho(r) \sin \rho(r)[1-\exp (-i \delta(r))] \\
\sin ^{2} \rho(r)+\cos ^{2} \rho(r) \exp (-i \delta(r))
\end{array}\right\| .
\end{aligned}
$$

Here, $\rho(r)$ is the angle (orientation) of the optical axis, which is determined by the direction of packing of birefringent protein fibrils with a transverse size $d(r) ; \quad \delta(r)=2 \pi / \lambda \Delta n d(r)$ - phase shift between the orthogonal components $\left(U_{0 x}, U_{0 y}\right)$ of amplitude $\left(U_{0}\right)$ of laser beam with the wavelength $\lambda$ at coordinate $r$.

Analytically, the process of transformation of the amplitude and phase of the laser beam by the crystal protein of the extracellular matrix in biological tissue by coordinates can be described using the equation

$$
\begin{gathered}
\left(\begin{array}{c}
U_{x}(r) \\
U_{y}(r)
\end{array}\right)=\left\|\begin{array}{cc}
d_{11}(r) & d_{12}(r) \\
d_{21}(r) & d_{22}(r)
\end{array}\right\|\left(\begin{array}{c}
U_{0 x} \\
U_{0 y} \exp \left(-i \delta_{0}\right)
\end{array}\right) \\
=\left(\begin{array}{c}
d_{11}(r) U_{0 x}+d_{12}(r) U_{0 y} \exp \left(-i \delta_{0}\right) \\
d_{21}(r) U_{0 x}+d_{22}(r) U_{0 y} \exp \left(-i \delta_{0}\right)
\end{array}\right) \\
=\left(\begin{array}{c}
d_{11}(r)+d_{12}(r) \operatorname{tg} \Omega_{0} \exp \left(-i \delta_{0}\right) \\
d_{21}(r)+d_{22}(r) \operatorname{tg} \Omega_{0} \exp \left(-i \delta_{0}\right)
\end{array}\right)
\end{gathered}
$$

Here, $\delta_{0}$ is the phase shift between the orthogonal components $U_{0 x}$ and $U_{0 y}$ of the illuminating laser beam amplitude, $t g \Omega_{0}=U_{0 y} / U_{0 x}$.

In accordance with the approach outlined in Ref. 17, for two-point $\left(r_{1}, r_{2}\right)$ generalization of the analytical description of the anisotropy parameters $\left(\rho_{1}\left(r_{1}\right), \rho_{2}\left(r_{2}\right)\right.$ and $\left.\delta_{1}\left(r_{1}\right), \delta_{2}\left(r_{2}\right)\right)$ of protein crystal network in the biological tissue extracellular matrix, let us use (6) and (7), taking into account Eq. (11)

$$
\begin{aligned}
& {\left[d_{11}\left(r_{1}\right)+d_{12}\left(r_{1}\right) t g \Omega_{0} \exp \left(-i \delta_{0}\right)\right]} \\
& \nu_{1}\left(d_{i k}\right)=\frac{\times\left[d_{11}\left(r_{2}\right)+d_{12}\left(r_{2}\right) t g \Omega_{0} \exp \left(-i \delta_{0}\right)\right]^{*}}{2} \\
& {\left[d_{21}\left(r_{1}\right)+d_{22}\left(r_{1}\right) t g \Omega_{0} \exp \left(-i \delta_{0}\right)\right]} \\
& -\frac{\times\left[d_{21}\left(r_{2}\right)+d_{22}\left(r_{2}\right) t g \Omega_{0} \exp \left(-i \delta_{0}\right)\right]^{*}}{2}, \\
& {\left[d_{11}\left(r_{1}\right)+d_{12}\left(r_{1}\right) \operatorname{tg} \Omega_{0} \exp \left(-i \delta_{0}\right)\right]} \\
& \nu_{2}\left(d_{i k}\right)=\frac{\times\left[d_{21}\left(r_{2}\right)+d_{22}\left(r_{2}\right) t g \Omega_{0} \exp \left(-i \delta_{0}\right)\right]^{*}}{2} \\
& {\left[d_{21}\left(r_{1}\right)+d_{22}\left(r_{1}\right) t g \Omega_{0} \exp \left(-i \delta_{0}\right)\right]} \\
& +\frac{\times\left[d_{11}\left(r_{2}\right)+d_{12}\left(r_{2}\right) t g \Omega_{0} \exp \left(-i \delta_{0}\right)\right]^{*}}{2}, \\
& \nu_{3}\left(d_{i k}\right)=i\left\{\begin{array}{c}
\frac{\left[d_{11}\left(r_{1}\right)+d_{12}\left(r_{1}\right) \operatorname{tg} \Omega_{0} \exp \left(-i \delta_{0}\right)\right]}{\times\left[d_{21}\left(r_{2}\right)+d_{22}\left(r_{2}\right) \operatorname{tg} \Omega_{0} \exp \left(-i \delta_{0}\right)\right]^{*}} \\
2 \\
\left.-\frac{\times\left[d_{21}\left(r_{1}\right)+d_{22}\left(r_{1}\right) \operatorname{tg} \Omega_{0} \exp \left(-i \delta_{0}\right)\right]}{2}\left(r_{2}\right)+d_{12}\left(r_{2}\right) \operatorname{tg} \Omega_{0} \exp \left(-i \delta_{0}\right)\right]^{*}
\end{array}\right\} .
\end{aligned}
$$

Substituting the coefficients of (12) into expression (6), we obtain the following expression 
for the parameter $W\left(r_{1}, r_{2}\right)$ that characterizes the degree of coordination between the orientation $\left(\rho_{1}\left(r_{1}\right), \rho_{2}\left(r_{2}\right)\right)$ - phase $\left(\delta_{1}\left(r_{1}\right) ; \delta_{2}\left(r_{2}\right)\right)$ parameters of anisotropy in various points $\left(r_{1}, r_{2}\right)$ of birefringent protein crystal network in the extracellular matrix

$$
\begin{aligned}
W\left(r_{1}, r_{2}\right)= & \frac{\left(A\left\{d_{i k}\left[\left(r_{1}, r_{2}\right)\right],\left[\delta_{2}\left(r_{2}\right)-\delta_{1}\left(r_{1}\right)\right], t g \Omega_{0}, \delta_{0}\right\}\right.}{C\left\{d_{i k}\left[\left(r_{1}, r_{2}\right)\right],\left[\delta_{2}\left(r_{2}\right)-\delta_{1}\left(r_{1}\right)\right], t g \Omega_{0}, \delta_{0}\right\}} \\
& +\frac{\left.B\left\{d_{i k}\left[\left(r_{1}, r_{2}\right)\right],\left[\delta_{2}\left(r_{2}\right)-\delta_{1}\left(r_{1}\right)\right], t g \Omega_{0}, \delta_{0}\right\}\right)^{2}}{C\left\{d_{i k}\left[\left(r_{1}, r_{2}\right)\right],\left[\delta_{2}\left(r_{2}\right)-\delta_{1}\left(r_{1}\right)\right], t g \Omega_{0}, \delta_{0}\right\}},
\end{aligned}
$$

where

$$
\begin{aligned}
& A=\left[d_{11}\left(r_{1}\right)+d_{12}\left(r_{1}\right) \operatorname{tg} \Omega_{0} \exp \left(-i \delta_{0}\right)\right] \\
& \times\left[d_{11}\left(r_{2}\right)+d_{12}\left(r_{2}\right) \operatorname{tg} \Omega_{0} \exp \left(-i \delta_{0}\right)\right]^{*}, \\
& B=\left\{[ d _ { 2 1 } ( r _ { 1 } ) + d _ { 2 2 } ( r _ { 1 } ) t g \Omega _ { 0 } \operatorname { e x p } ( - i \delta _ { 0 } ) ] \left[d_{21}\left(r_{2}\right)\right.\right. \\
& \left.\left.+d_{22}\left(r_{2}\right) \operatorname{tg} \Omega_{0} \exp \left(-i \delta_{0}\right)\right]^{*}\right\} \\
& \times \exp \left\{-i\left[\delta_{2}\left(r_{2}\right)-\delta_{1}\left(r_{1}\right)\right]\right\} \text {, } \\
& C=\left\{\begin{array}{c}
{\left[d_{11}\left(r_{1}\right)+d_{12}\left(r_{1}\right) \operatorname{tg} \Omega_{0} \exp \left(-i \delta_{0}\right)\right]} \\
\times\left[d_{11}\left(r_{1}\right)+d_{12}\left(r_{1}\right) \operatorname{tg} \Omega_{0} \exp \left(-i \delta_{0}\right)\right]^{*} \\
+\left[d_{21}\left(r_{1}\right)+d_{22}\left(r_{1}\right) \operatorname{tg} \Omega_{0} \exp \left(-i \delta_{0}\right)\right] \\
\times\left[d_{21}\left(r_{1}\right)+d_{22}\left(r_{1}\right) \operatorname{tg} \Omega_{0} \exp \left(-i \delta_{0}\right)\right]^{*}
\end{array}\right\} \\
& +\left\{\begin{array}{c}
{\left[d_{11}\left(r_{2}\right)+d_{12}\left(r_{2}\right) \operatorname{tg} \Omega_{0} \exp \left(-i \delta_{0}\right)\right]} \\
\times\left[d_{11}\left(r_{2}\right)+d_{12}\left(r_{2}\right) \operatorname{tg} \Omega_{0} \exp \left(-i \delta_{0}\right)\right]^{*} \\
+\left[d_{21}\left(r_{2}\right)+d_{22}\left(r_{2}\right) t g \Omega_{0} \exp \left(-i \delta_{0}\right)\right] \\
\times\left[d_{21}\left(r_{2}\right)+d_{22}\left(r_{2}\right) t g \Omega_{0} \exp \left(-i \delta_{0}\right)\right]^{*}
\end{array}\right\} .
\end{aligned}
$$

Like in Ref. 17, in what follows we shall call the parameter $W\left(r_{1}, r_{2}\right)$ as the complex degree of mutual anisotropy (CDMA) of biological tissues.

Our analysis of Eqs. (11)-(16) shows the dependence of orthogonal components $U_{x}(r), U_{y}(r)$ of the complex amplitude on rotation of the polarization plane $\left(\Omega_{0}\right)$ of the illuminating laser wave or rotation $(\gamma)$ of the biological tissue layer to the irradiation direction. The comparative diagnostic analysis of CDMA in extracellular matrix of various biological tissues according to it becomes more difficult. The only exception exists in the case of irradiation of biological tissues by the circular polarized laser wave $U_{0}$

$$
U_{0}=\left(\begin{array}{c}
U_{0 x} \\
U_{0 y}
\end{array}\right) \equiv \frac{1}{\sqrt{2}}\left(\begin{array}{l}
1 \\
i
\end{array}\right) .
$$

Therefore, to determine the analytical expression for CDMA $W\left(r_{1}, r_{2}\right)$ in two points $\left(r_{1}, r_{2}\right)$ of the extracellular matrix of the biological tissues, we need to use the relations (5) and (7) and take into account Eqs. (8) and (9)

$$
\begin{aligned}
& W\left(r_{1}, r_{2}\right) \\
& \quad\left\{\left[d_{11}\left(r_{1}\right)+i d_{12}\left(r_{1}\right)\right]\left[d_{11}\left(r_{2}\right)+i d_{12}\left(r_{2}\right)\right]^{*}\right. \\
& =\frac{\left.+\left[d_{21}\left(r_{1}\right)+i d_{22}\left(r_{1}\right)\right]\left[d_{21}\left(r_{2}\right)+i d_{22}\left(r_{2}\right)\right]^{*}\right\}^{2}}{I\left(r_{1}\right) I\left(r_{2}\right)} .
\end{aligned}
$$

Here, $I\left(r_{1}\right)$ and $I\left(r_{2}\right)$ are defined in accordance to relations

$$
\begin{aligned}
I\left(r_{1}\right)= & \left\{\left[d_{11}\left(r_{1}\right)+i d_{12}\left(r_{1}\right)\right]\left[d_{11}\left(r_{1}\right)+i d_{12}\left(r_{1}\right)\right]^{*}\right. \\
& \left.+\left[d_{21}\left(r_{1}\right)+i d_{22}\left(r_{1}\right)\right]\left[d_{21}\left(r_{1}\right)+i d_{22}\left(r_{1}\right)\right]^{*}\right\}, \\
I\left(r_{2}\right)= & \left\{\left[d_{11}\left(r_{2}\right)+i d_{12}\left(r_{2}\right)\right]\left[d_{11}\left(r_{2}\right)+i d_{12}\left(r_{2}\right)\right]^{*}\right. \\
& \left.+\left[d_{21}\left(r_{2}\right)+i d_{22}\left(r_{2}\right)\right]\left[d_{21}\left(r_{1}\right)+i d_{22}\left(r_{2}\right)\right]^{*}\right\} .
\end{aligned}
$$

Taking the real part $\left(\operatorname{Re}\left\{d_{i k}(r)\right\} \equiv \tilde{d}_{i k}(r)\right)$ of the Jones matrix elements (Eq. (18)), we obtain an expression for the real part of the CDMA $\left(\operatorname{Re}\left\{W\left(r_{1}, r_{2}\right)\right\} \equiv \tilde{W}\left(r_{1}, r_{2}\right)\right)$ for the extracellular matrix of a biological tissue layer, which can be obtained by direct polarization measurements

$$
\begin{aligned}
& \tilde{W}\left(r_{1}, r_{2}\right)= \\
& \quad\left\{\left[\tilde{d}_{11}\left(r_{1}\right) \tilde{d}_{11}\left(r_{2}\right)-\tilde{d}_{12}\left(r_{1}\right) \tilde{d}_{12}\left(r_{2}\right)\right]\right. \\
& \quad \frac{\left.+\left[\tilde{d}_{21}\left(r_{1}\right) \tilde{d}_{21}\left(r_{2}\right)-\tilde{d}_{22}\left(r_{1}\right) \tilde{d}_{22}\left(r_{2}\right)\right]\right\}^{2}}{I\left(r_{1}\right) I\left(r_{2}\right)},
\end{aligned}
$$

where

$$
\left\{\begin{array}{l}
\tilde{d}_{11}(r)=\cos ^{2} \rho(r)+\sin ^{2} \rho(r) \cos \delta(r), \\
\tilde{d}_{12,21}(r)=\cos \rho(r) \sin \rho(r)(1-\cos \delta(r)), \\
\tilde{d}_{22}(r)=\sin ^{2} \rho(r)+\cos ^{2} \rho(r) \cos \delta(r) .
\end{array}\right.
$$

Let us analyze the relationship of CDMA $\tilde{W}\left(r_{1}, r_{2}\right)$ of biological tissue layer extracellular matrix with various orientations $\rho_{1}\left(r_{1}\right), \rho_{2}\left(r_{2}\right)$ of optical axes and the phase shifts $\delta_{1}\left(r_{1}\right), \delta_{2}\left(r_{2}\right)$ that are introduced by protein crystals of the extracellular matrix in the points $\left(r_{1}, r_{2}\right)$. Table 1 shows the main characteristic values of CDMA $\tilde{W}\left(r_{1}, r_{2}\right)$ in two arbitrary points $\left(r_{1}, r_{2}\right)$ of the extracellular matrix of biological tissue layer. For comparison, the right side shows the types of polarization 
Table 1. Characteristic values of CDMA $\tilde{W}\left(r_{1}, r_{2}\right)$ of the biological tissue extracellular matrix and of CDMP $\tilde{V}\left(r_{1}, r_{2}\right)$ of corresponding laser images.

\begin{tabular}{lccc}
\hline $\begin{array}{l}\text { The range of } \rho\left(r_{1}\right), \rho\left(r_{2}\right) \\
\text { changing, }(0 \div \pi)\end{array}$ & $\begin{array}{c}\text { The range of } \delta\left(r_{1}\right)=\delta\left(r_{2}\right) \\
\text { changing, }(0 \div \pi)\end{array}$ & $\begin{array}{c}\text { The value of } \\
\tilde{W}\left(r_{1}, r_{2}\right)\end{array}$ & $\begin{array}{c}\text { The value of } \\
\tilde{V}\left(r_{1}, r_{2}\right)\end{array}$ \\
\hline$\rho\left(r_{1}\right)=\rho\left(r_{2}\right)$, & 0 & 1.0 & 1.0 \\
$\rho\left(r_{2}\right)=0.5 \pi+\rho\left(r_{2}\right)$ & $0.5 \pi$ & 1.0 & 1.0 \\
& $\pi$ & 1.0 & 1.0 \\
$\rho\left(r_{1}\right)=\rho\left(r_{2}\right)$, & $\delta_{2}\left(r_{1}\right)=0.5 \pi+\delta_{1}\left(r_{2}\right)$ & $\tilde{W}\left(r_{1}, r_{2}\right)$ & $\tilde{V}\left(r_{1}, r_{2}\right)$ \\
$\rho\left(r_{2}\right)=0.5 \pi+\rho\left(r_{2}\right)$ & $0.5 \pi$ & 0.5 & 0.5 \\
& $\pi$ & 0.5 & 0.5 \\
& $1.5 \pi$ & 0.5 & 0.5 \\
$\rho\left(r_{1}\right)=\rho\left(r_{2}\right)$, & $\delta_{2}\left(r_{1}\right)=\pi+\delta_{1}\left(r_{2}\right)$ & $\tilde{W}\left(r_{1}, r_{2}\right)$ & $\tilde{V}\left(r_{1}, r_{2}\right)$ \\
$\rho\left(r_{2}\right)=0.5 \pi+\rho\left(r_{2}\right)$ & $\pi$ & 0 & 0 \\
& $1.5 \pi$ & 0 & 0 \\
& $2 \pi$ & 0 & 0 \\
\hline
\end{tabular}

$\left(U_{x}, U_{y}, \delta\right)$ of light fluctuations in the sample points of biological tissue laser images and the corresponding values of CDMP $\tilde{V}\left(r_{1}, r_{2}\right)$.

Analysis of the presented data shows that in the case of single scattering of laser radiation, there is a unique relationship between the characteristic values of CDMA $\tilde{W}\left(r_{1}, r_{2}\right)$ of biological tissue layer and CDMP $\tilde{V}\left(r_{1}, r_{2}\right)$ of biological tissue laser images. On the other hand, with increase of multiplicity of light scattering or the propagation in free space of differently polarized coherent waves $U\left(r_{1}\right)$ and $U\left(r_{2}\right)$ their cross-interference will have a place. As a result, the phase shifts $\left(\delta^{*}\right)$ and the ratio between the orthogonal components $\left(U_{x}^{*}, U_{y}^{*}\right)$ of the net fluctuation amplitudes in the points $r_{1}, r_{2}$ are changed.

Therefore, because of interference averaging, the unambiguity in inter-relations between the CDMP and the object structure is eliminated. At the same time, the magnitude of CDMA parameter is determined only by the biological tissue anisotropy. That is why, the task of searching the comparative diagnostic efficiency of both the methods in such conditions appears to be topical.

\section{Optical Scheme and Experimental Measurements of Two-Dimensional CDMA Distributions of Biological Tissues}

Experimental investigations were performed using the classical polarimeter, the main parts and elements of which have been presented in Fig. $1 .^{19}$

It was illuminated by collimated $\left(\varnothing=10^{4} \mu \mathrm{m}\right)$ He-Ne laser beam $(\lambda=0.6328 \mu \mathrm{m})$ with the power close to $50 \mu \mathrm{W}$. The polarization illuminator (quarter-wave plates 3, 5 and polarizer 4) formed the beam with an arbitrary polarization azimuth $\left(0^{\circ} \leq \alpha_{0} \leq 180^{\circ}\right)$ and ellipticity $\left(0^{\circ} \leq \beta_{0} \leq 90^{\circ}\right)$. Polarization images of biological tissues by means of the micro-objective 7 (focal distance $-1.5 \mathrm{~cm}$, aperture -0.2 , magnification $-4 \times$ ) were projected into the plane of light-sensitive area of a CCD

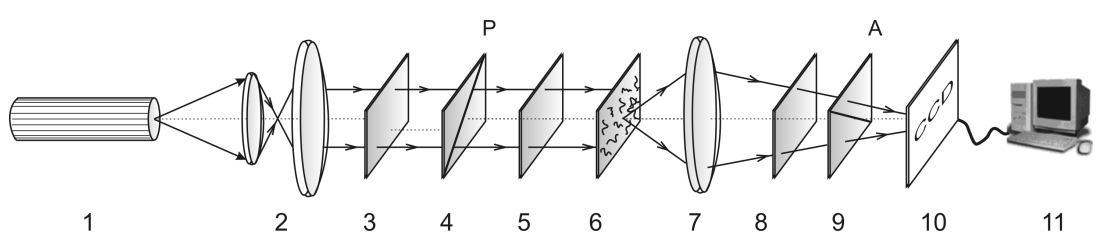

Fig. 1. Optical scheme of the polarimeter for measuring the coordinate CDMA distributions inherent to biological tissues. 1 He-Ne laser $(\lambda=0.6328 \mu \mathrm{m}) ; 2$ - collimator; 3,5 and 8 - quarter-wave plates; 4 and 9 polarizers; 6 - biological tissue histological section; 7 - projection micro-objective; 10 - CCD camera; 11 - PC. 
camera (overall amount of pixels $-800 \times 600$, light sensitive area size $-4000 \times 3000 \mu \mathrm{m}$, deviation of photosensitive characteristics from linear ones was no more than 15\%), which provided the range of measuring the structural elements of biological tissue with the resolution $2-2000 \mu \mathrm{m}$. Maximal resolution verification $(2 \mu \mathrm{m})$ was performed using the stage micrometer (linear scale), and that image was projected into the light sensitive area of the CCD camera by using the micro-objective 7 .

The minimal resolution $(2000 \mu \mathrm{m})$ corresponds to the situation when the light sensitive area of the CCD camera is entirely filled by two equal sized structural elements (light and dark) of the stage micrometer. The conditions of the experiment were chosen in such a way that it enabled to reduce the space-angular aperture filtering while forming the biological tissue images. This was ensured by conformance of angular characteristics of indicatrices of light scattering by the biological tissue samples $\left(\Omega \approx 16^{\circ}\right)$ and angular aperture of the micro-objective $\left(\Delta \omega=20^{\circ}\right)$. Here, $\Omega$ is the solid angle within which $98 \%$ of all the energy of scattered radiation is concentrated.

In order to determine the coordinate distribution of CDMP parameter (9), the following technique was used:

(1) In the absence of polarization filter 8, 9 (Fig. 1), the intensity coordinate distribution $\left(\begin{array}{c}I\left(r_{11}\right), \ldots, I\left(r_{1 n}\right) \\ I\left(r_{m 1}\right), \ldots, I\left(r_{m n}\right)\end{array}\right)$ of the points of biological tissue layer image was measured;

(2) Rotating the transmission plane of polarizer 9 (Fig. 1) by the angles $\Theta=0^{\circ} ; \Theta=90^{\circ}$, the following coordinate distributions were measured: $\left(\begin{array}{c}I_{x}\left(r_{11}\right), \ldots, I_{x}\left(r_{1 n}\right) \\ I_{x}\left(r_{m 1}\right), \ldots, I_{x}\left(r_{m n}\right)\end{array}\right)$ and $\left(\begin{array}{c}I_{y}\left(r_{11}\right), \ldots, I_{y}\left(r_{1 n}\right) \\ I_{y}\left(r_{m 1}\right), \ldots, I_{y}\left(r_{m n}\right)\end{array}\right)$;

(3) Making further rotation of the analyzer 9 transmission plane $(\Theta)$ within the limits $\Theta=0^{\circ}-180^{\circ}$ allows to determine minimum $\left(I_{\min }\left(r_{j k}\right)\right)$ and maximum $\left(I_{\max }\left(r_{j k}\right)\right)$ levels of the intensity for each pixel $\left(r_{j k}\right)$ of the CCD camera. Thus, the arrays of the intensity extreme values $I_{\min }(m \times n), I_{\max }(m \times n)$ of laser images of biological tissue histological Sec. 6 and the corresponding angles of rotation $\Theta_{\min }(m \times n) \Leftrightarrow I_{\min }(m \times n)$ of the analyzer 9 can be obtained;

(4) On the basis of the obtained data, it is possible to calculate the coordinate distribution of the azimuth $\alpha(m \times n)$ and ellipticity $\beta(m \times n)$ of polarization and determine the two-dimensional distribution of phase shifts by using the following algorithms

$$
\left\{\begin{array}{l}
\alpha(r)=0,5 \pi-\Theta(r), \\
\beta(r)=\frac{\operatorname{arctg} I_{\min }(r)}{I_{\max }(r)}, \\
\delta(r)=\operatorname{arctg}\left[\frac{\operatorname{tg} 2 \beta(r)}{t g \alpha(r)}\right]
\end{array}\right.
$$

(5) For each pair of points $r_{j, k}-r_{j+1, k}$, using the algorithm (9), the following value of CDMP parameter $\tilde{V}\left(r_{j, k}, r_{j+1, k}\right)$ was calculated;

(6) This operation was repeated for each line of the studied image by using the one-pixel linear shift.

The coordinate distribution of CDMA parameter was determined in the same manner:

(1) Within the limits of each pixel, using the classic technique $^{4}$ the set of Jones matrix elements $d_{i k}(r)$ was calculated;

(2) On the grounds of matrix elements $d_{i k}(r)$ and phase shift $\delta(r)$, the value of CDMA parameter $\tilde{W}\left(r_{j, k}, r_{j+1, k}\right)$, was calculated for each pair of points $r_{j, k}-r_{j+1, k}$ by using the algorithm (12);

(3) This operation was repeated for each line of the image by using the one-pixel linear shift.

\section{Experimental Researches of Two-Dimensional Distribution of CDMA of Biological Tissues Being in Different Morphological and Physiological States}

It should be noted that the structure of the anisotropic component of the different types of biological tissues is rather complicated and diverse. ${ }^{18}$ Therefore, the analysis of two-dimensional distribution of optical parameters corresponding to the biological tissue extracellular matrix of various types objectively requires complex statistical, correlation, fractal and topological ${ }^{4}$ as well as other approaches. In our work, we will restrict ourselves by studying the inter-relation of statistical moments of the 1st to 4 th orders, ${ }^{21}$ which characterize the coordinate distributions $\tilde{W}(x, y)$ of the biological tissue extracellular matrix, with pathological changes in its orientation-phase structure. 




(a)

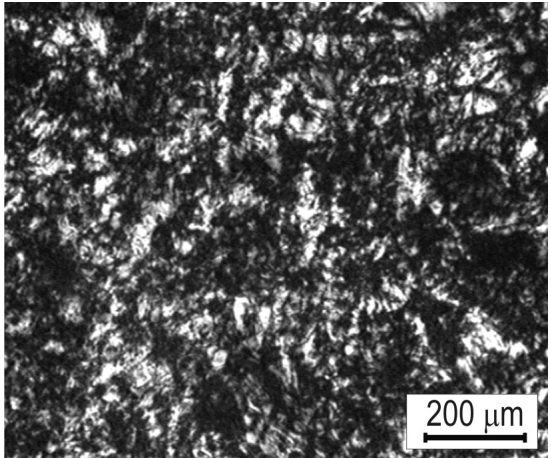

(b)

Fig. 2. Architectonics of the muscular tissue (a) and dermal layer (b) in crossed polarizer and analyzer.

As objects for investigations (Fig. 2), we chose histological sections of myocardium muscle tissue (MT) [Fig. 2(a)] and skin derma (SD) layer [Fig. 2(b)]. This choice of objects was due to the fact that the birefringence indexes $(\Delta n)$ of myosin $[\mathrm{MT}-\Delta n=$ $1.35 \times 10^{-2}$ (see Ref. 23)] and collagen [SD $-\Delta n=$ $1.5 \times 10^{-2}$ (see Ref. 24)] fibrils of the extracellular matrix of these tissues are similar. Therefore, we can assume that the ranges of phase shifts values $(\delta(m \times n))$ are determined primarily by transverse geometric dimensions $d(m \times n)$ of protein crystals. The range of sizes for a network of SD collagen fibrils comprises the following interval $d=5-45 \mu \mathrm{m}$. For a MT layer of myosin fibrils, variations of sizes lie within the range $d=25-35 \mu \mathrm{m}$.

Pathological changes of the MT morphological structure (myocardial dystrophy) are manifested in the reduction of birefringence and disordering of packing directions in the network of myosin fibrils. The peculiarity of the extracellular matrix structure of healthy SD is a random distribution of directions $(\rho(x \times y))$ of the protein crystal optical axes (Fig. 2). The extracellular matrix in oncologically changed samples is characterized by a set of ordered "the newly formed" collagen fibrils with a greater value of the birefringence parameter. ${ }^{25}$

Therefore, comparative studies of CDMA coordinate distributions for this biological tissue extracellular matrix will help to determine the range of statistical parameter variations $\tilde{W}(x, y)$ and on this basis, to implement the diagnostics of their physiological state.

The series of coordinate distributions $[600 \mathrm{pix} \times$ 800 pix — fragments (a), (d); 50 pix $\times 50$ pix fragments (b), (e)] and the histograms [fragments (c), (f)] of CDMA values $\tilde{W}(x, y)$ of physiologically normal [fragments (a), (b), (c)] and pathologically changed [fragments (d), (e), (f)] histological sections of MT (Fig. 3) and SD (Fig. 4) histological sections.

The analysis of the obtained experimental data has revealed that the coordinate distributions of CDMA [Figs. 3(a), 3(d) and 4(a), 4(d)] are formed by the set of the local areas [domains $\tilde{W} \approx$ const. Figs. 3(b), 3(e) and 4(b), 4(e)] with the maximum possible range of variations $0 \leq \tilde{W} \leq 1$ [Figs. $3(\mathrm{c})$, $3(\mathrm{f})$ and $4(\mathrm{c}), 4(\mathrm{f})]$.

The two-dimensional distributions [Figs. 3(a), $3(\mathrm{~d})$ ] of CDMA $\tilde{W}(x, y)$ of MT samples are formed by the domains [Fig. 3(b), 3(e)] with the sizes $10-30 \mu \mathrm{m}$. Degenerative changes in myosin fibril network appear in the process of the scale reducing $(15 \%-30 \%)$ of the domain structure of CDMA coordinate distribution.

The coordinate structure of CDMA parameter $\tilde{W}(x, y)$ in the SD collagen network is small-scale [Figs. 4(a), 4(d)]. The range of geometric dimensions in the domain $\tilde{W}\left(x_{i}, y_{i}\right) \approx$ const. changes [Figs. 4(b), 4(e)] varies within the limits 5-15 $\mu \mathrm{m}$. Cancer changes of SD architectonic network are revealed in some increase (up to $10 \%-15 \%$ ) of the local domains scale.

The experimental data satisfactorily correlate with the proposed model analysis of the extracellular matrix structure with the help of CDMA. Thus, there will always be points $(r, r+\Delta r)$ in the network of protein fibrils that define such relationships between the parameters of anisotropy $(\rho, \delta)$ (Table 1), which correspond to extreme values of

$$
\text { CDMA } \tilde{W}(r, r+\Delta r)=\left\{\begin{array}{l}
0 \\
1
\end{array}\right.
$$






(a)

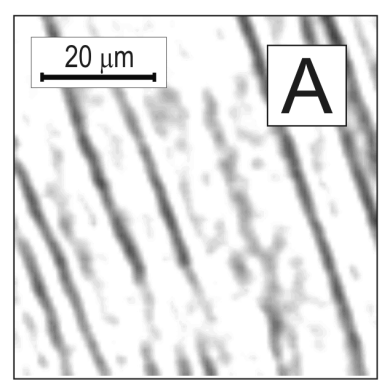

(b)



(c)

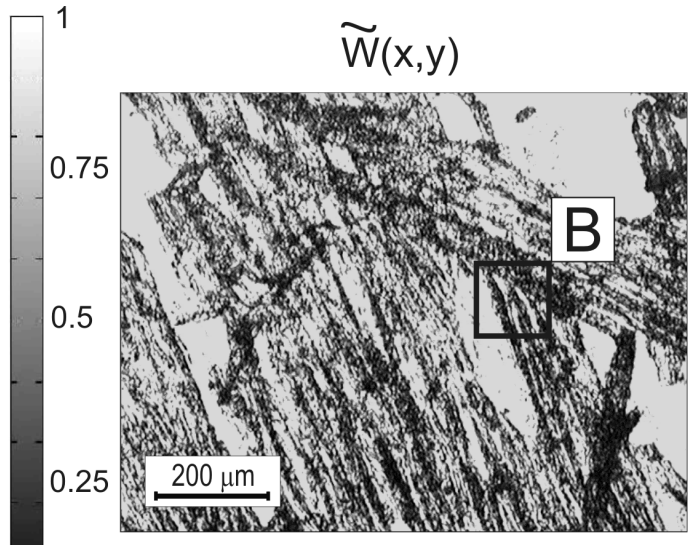

(d)

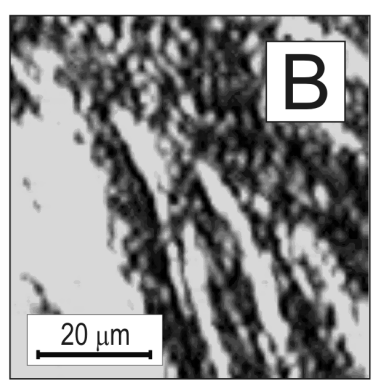

(e)

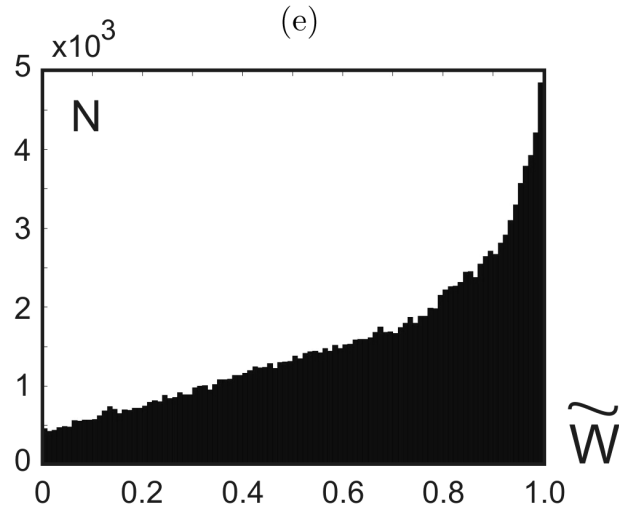

(f)

Fig. 3. Coordinate distributions $[600$ pix $\times 800$ pix — fragments (a), (d); 50 pix $\times 50$ pix — fragments (b), (e)] and histograms [fragments (c), (f)] of values $\tilde{W}(x, y)$ for physiologically normal [(a), (b), (c)] and pathologically changed [(d), (e), (f)] MT.

On the other hand, the specificity of the morphological construction of biological tissue extracellular matrix of various types manifests itself in different probabilities of CDMA $\tilde{W}(x, y)$ values. It is believed that, for directional ordered $(\rho(x, y))$ and the geometric dimensions $(d(x, y))$ of MT myosin fibril network [Fig. 3(a)], the dispersion of orientations of optical axes and the phase shifts are substantially less than for the disordered network of SD collagen fibrils [Fig. 4(a)]. Therefore, the distribution of CDMA random values of MT layer is predominantly localized in a small range $(0.5 \leq$ $W \leq 1)$ relatively to the main extremes [Fig. 3(c)]. Histograms of CDMA values $\tilde{W}(x, y)$ of SD layer are fairly equiprobable distribution in the widest possible range $(0 \leq W \leq 1)$ of changing this parameter [Fig. 4(c)].

Degenerative changes of MT myosin crystal network are shown [Fig. 3(e)] in the distribution of extreme CDMA values $(\tilde{W} \rightarrow 1)$ to lower values 


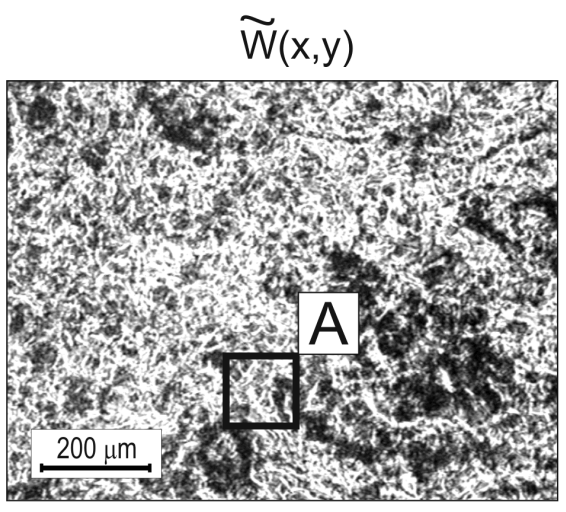

(a)

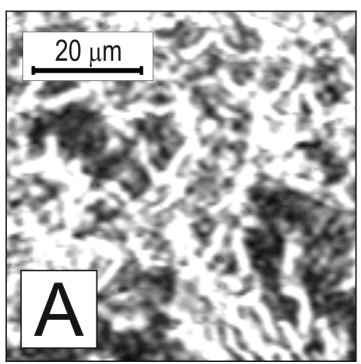

(b)

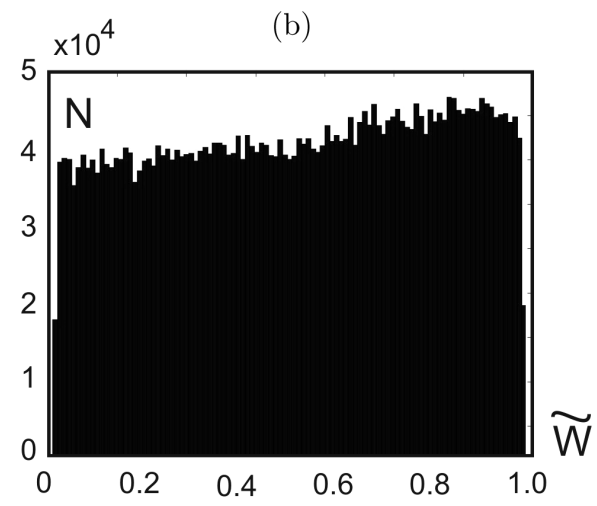

(c)

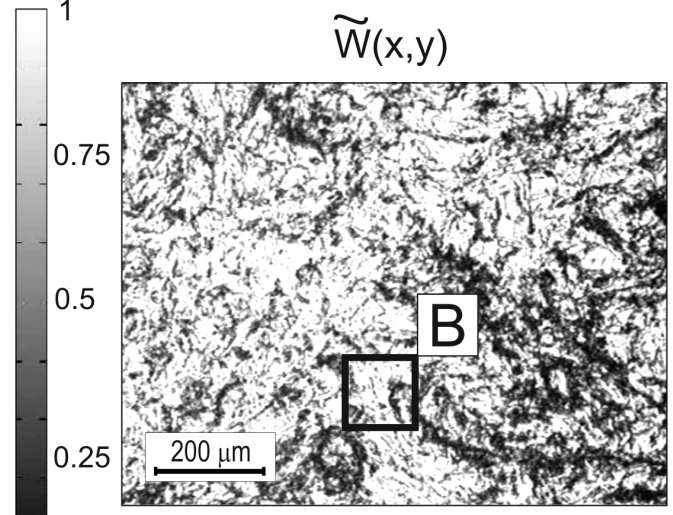

(d)



(e)



(f)

Fig. 4. Coordinate distributions $[600$ pix $\times 800$ pix - fragments (a), (d); 50 pix $\times 50$ pix — fragments (b), (e)] and histograms [fragments (c), (f)] of values $\tilde{W}(x, y)$ for physiologically normal $[(\mathrm{a}),(\mathrm{b}),(\mathrm{c})]$ and oncologically changed [(d), (e), (f)] SD tissues.

$(\tilde{W}=0.5-0.8)$. Early oncological changes of SD are accompanied with the formation of a direction for collagen fibril growth. It is optically shown [Fig. 4(e)] in some localization of the CDMA random values distribution [Fig. 4(f)] in the domain of $\tilde{W}=0.4-0.6$ extremes.

In order to obtain some objective criteria of diagnostic efficiency, the comparative investigation of CDMP $(\tilde{V}(x, y))$ (see Ref. 9) and CDMA $\tilde{W}(x, y)$ techniques was performed in the conditions of single and multiple scattering of laser radiation by the layers of MT and SD.
5. Comparative Statistical Analysis of Two-Dimensional Distributions of CDMA of Biological Tissues Extracellular Matrix and CDMP of its Laser Images

With the aim to determine the ranges of statistical moment changes that characterize the coordinate distributions of $\tilde{W}(x, y)$ and $\tilde{V}(x, y)$, four groups of histological sections of MT (healthy 21 samples; dystrophically changed - 19 samples) and SD (healthy — 20 samples; oncologically 







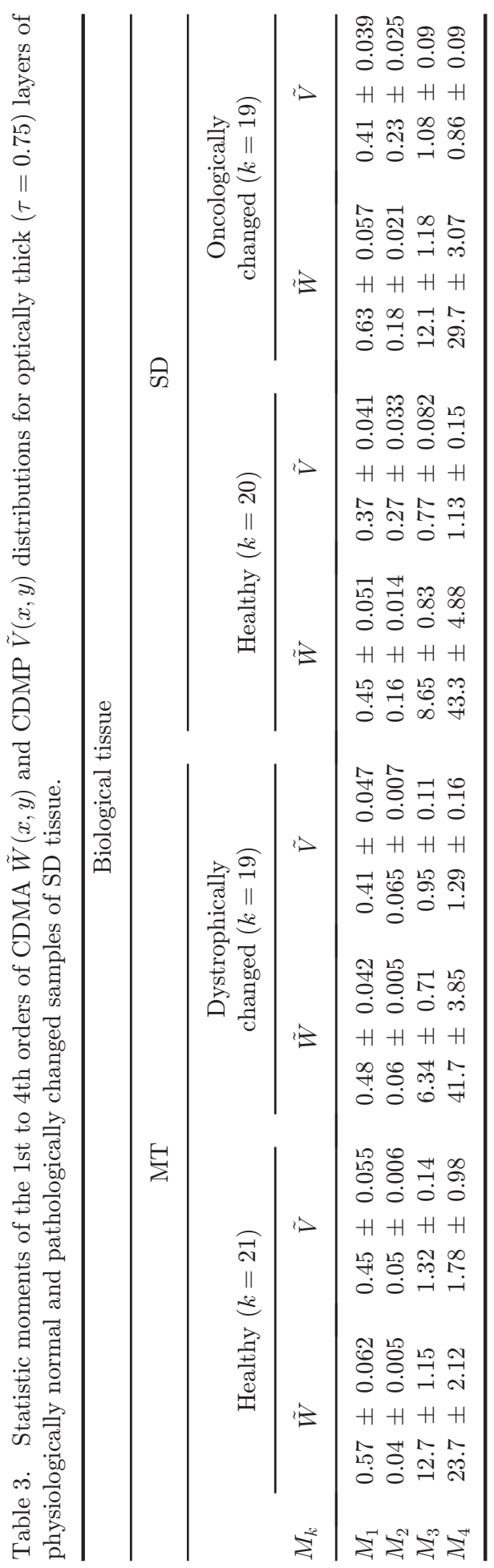


changed — 19 samples) layer samples were formed.

Statistical moments of the 1 st to 4 th orders $M_{k=1,2,3,4}$ for $Q=\left\{\begin{array}{l}\tilde{V} \\ \tilde{W}\end{array}\right.$ distributions were calculated using the Matlab software product based on the following algorithms ${ }^{21}$

$$
\begin{array}{ll}
M_{1}=\frac{1}{N} \sum_{i=1}^{N}\left|Q_{i}\right|, & M_{2}=\sqrt{\frac{1}{N} \sum_{i=1}^{N} Q_{i}^{2}}, \\
M_{3}=\frac{1}{M_{2}^{3}} \frac{1}{N} \sum_{i=1}^{N} Q_{i}^{3}, & M_{4}=\frac{1}{M_{2}^{4}} \frac{1}{N} \sum_{i=1}^{N} Q_{i}^{4} .
\end{array}
$$

where $N=m \times n$ is the amount of pixels in the CCD-camera photosensitive area.

In Tables 2 and 3, the comparative results of calculations of the average $\left(M_{1}\right)$, the dispersion $\left(M_{2}\right)$, the skewness $\left(M_{3}\right)$ and the kurtosis $\left(M_{4}\right)$ of CDMA $\tilde{W}(x, y)$ distributions of four groups for MT and SD with different optical thicknesses (extinction coefficients $\tau=0.09$ and $\tau=0.75$ ) and of CDMP $\tilde{V}(x, y)$ for their laser images are presented.

From the obtained data about the coordinate distributions of CDMA for optically thin $(\tau=0.09)$ layers of MT and SD tissues, one can see that:

- The average and dispersion of distributions $\tilde{W}(x, y)$ for physiologically normal and pathologically changed MT and SD differ insufficiently within $1.3\left(M_{1}\right)-1.5\left(M_{2}\right)$ times. For two-dimensional distributions $\tilde{V}(x, y)$ of laser images, there is practically no difference between $M_{1}$ and $M_{2}$.

- The skewness values $M_{3}$ of distributions $\tilde{W}(x, y)$ of the investigated samples differ by 2.1 times and the kurtosis values by 3.2 times. For CDMP distributions $\tilde{V}(x, y)$, the values of the 3 rd and 4 th statistic moments vary for $M_{3}$ by 1.3 times and for $M_{4}$ by 1.8 times.

On the other hand, increase in the light scattering due to cross-interference of differently polarized coherent waves $U_{j}(r)$ changes the distribution of azimuths and ellipticities of polarization inherent to the biological tissue object field. As a result, the inter-relation between the parameters $\tilde{W}\left(r_{1}, r_{2}\right)$ and $\tilde{V}\left(r_{1}, r_{2}\right)$ is violated (Table 3 ):

- As diagnostically actual parameters of the physiological state of optically thick biological tissues, the statistical moments of higher orders can be used. Thus, skewness values $M_{3}$ of coordinate distributions $\tilde{W}(x, y)$ for healthy and oncologically changed SD tissues differ by 1.4 times and the kurtosis values $M_{4}$ by 1.45 times.

- The values of the 3rd- to 4th-order statistic moments of distributions $\tilde{V}(x, y)$ of CDMP for the object field of optically thick biological tissue layers do not depend much on their physiological state and are one order less than the values of the CDMP $\tilde{W}(x, y)$ skewness and kurtosis.

\section{Conclusion}

To characterize the degree of consistency of parameters of the optically uniaxial birefringent protein-fibril networks of biological tissues, the new parameter - CDMA has been offered. The technique of polarization measuring the coordinate distributions of the CDMA in biological tissues is developed. It has been shown that statistic approach to the analysis of CDMA distributions for biological tissues in various morphological and physiological states and for different optical thicknesses appears to be more sensitive and efficient in differentiation of physiological state in comparison with investigations of CDMP for corresponding laser images.

\section{References}

1. M. S. Patterson, S. Andersson-Engels, B. C. Wilson, E. K. Osei, "Absorption-spectroscopy in tissuesimulating materials - A theoretical and experimental-study of photon paths," Appl. Opt. 34, 22-30 (1995).

2. R. R. Alfano, J. G. Fujimoto, Eds., Advances in Optical Imaging and Photon Migration, Topics in Optics and Photonics Series, Vol. 2, Optical Society of America, Washington, DC (1996).

3. S. Bartel, A. H. Hielscher, "Monte Carlo simulation of the diffuse backscattering Mueller matrix for highly scattering media," Appl. Opt. 39, 1580-1588 (2000).

4. S. P. Morgan, M. P. Khong, M. G. Somekh, "Effects of polarization state and scatterer concentration on optical imaging through scattering media," Appl. Opt. 36, 1560-1565 (1997).

5. S. G. Demos, R. R. Alfano, "Optical polarization imaging," Appl. Opt. 36, 150-155 (1997).

6. S. L. Jacques, J. R. Roman, K. Lee, "Imaging superficial tissues with polarized light," Lasers Surg. Med. 26, 119-129 (2000). 
7. M. Born, E. Wolf, Principles of Optics, Cambridge Univ. Press, Cambridge, 1999.

8. A. G. Ushenko, V. P. Pishak, "Laser polarimetry of biological tissues, principles and applications," in Coherent-Domain Optical Methods: Biomedical Diagnostics, Environmental and Material Science, V. Tuchin, Ed., pp. 67-93, Kluwer Academic Publishers (2004).

9. F. Gori, M. Santarsiero, S. Vicalvi, R. Borghi, G. Guattari, "Beam coherence-polarization matrix," Pure Appl. Opt. 7, 941-951 (1998).

10. E. Wolf, "Unified theory of coherence and polarization of random electromagnetic beams," Phys. Lett. A 312, 263-267 (2003).

11. J. Tervo, T. Setala, A. Friberg, "Degree of coherence for electromagnetic fields," Opt. Express 11, 1137-1143 (2003).

12. J. M. Movilla, G. Piquero, R. Martínez-Herrero, P. M. Mejías, "Parametric characterization of nonuniformly polarized beams," Opt. Commun. 149, 230-234 (1998).

13. C. Mujat, A. Dogariu, "Statistics of partially coherent beams: A numerical analysis," J. Opt. Soc. Am. A 21(6), 1000-1003 (2004).

14. F. Gori, "Matrix treatment for partially polarized, partially coherent beams," Opt. Lett. 23, 241-243 (1998).

15. M. Mujat, A. Dogariu, "Polarimetric and spectral changes in random electromagnetic fields," Opt. Lett. 28, 2153-2155 (2003).

16. J. Ellis, A. Dogariu, S. Ponomarenko, E. Wolf, "Interferometric measurement of the degree of polarization and control of the contrast of intensity fluctuations," Opt. Lett. 29, 1536-1538 (2003).

17. J. Ellis, A. Dogariu, "Complex degree of mutual polarization," Opt. Lett. 29, 536-538 (2004).
18. S. C. Cowin, "How is a tissue built?" J. Biomech. Eng. 122, 553-568 (2000).

19. O. V. Angelsky, A. G. Ushenko, Ye. G. Ushenko, "Complex degree of mutual polarization of biological tissue coherent images for the diagnostics of their physiological state," J. Biomed. Opt. 10(6), 060502 (2005).

20. Ye. G. Ushenko, "Complex degree of mutual polarization of biotissue's speckle-images," Ukr. J. Phys. Opt. 6(3), 104-113 (2005).

21. Yu. A. Ushenko, "Statistical structure of polarization-inhomogeneous images of biotissues with different morphological structures," Ukr. J. Phys. Opt. 6(2), 63-70 (2005).

22. O. V. Angelsky, A. G. Ushenko, V. P. Pishak, D. N. Burkovets, S. B. Yermolenko, O. V. Pishak, Y. A. Ushenko, "Polarizing-correlative processing of images of statistic objects in visualization and topology reconstruction of their phase heterogeneity," Proc. SPIE 4016, 419-424 (1999).

23. O. V. Angelsky, D. N. Burkovets, V. P. Pishak, Yu. A. Ushenko, "Polarization-correlation investigations of biotissue multifractal structures and their pathological changes diagnostics," Laser Phys. 10(5), 1136-1142 (2000).

24. A. G. Ushenko, "The vector structure of laser biospeckle fields and polarization diagnostics of collagen skin structures," Laser Phys. 10(5), 1143-1149 (2000).

25. O. V. Angelsky, A. G. Ushenko, S. B. Ermolenko, D. N. Burkovets, V. P. Pishak, Yu. A. Ushenko, O. V. Pishak, "Polarization-based visualization of multifractal structures for the diagnostics of pathological changes in biological tissues," Opt. Spectrosc. 89(5), 799-804 (2000). 